\title{
The Snyder Classification of Superior Labrum Anterior and Posterior (SLAP) Lesions
}

\author{
Zahab S. Ahsan MD, Jason E. Hsu MD, Albert O. Gee MD
}

Received: 8 October 2015/ Accepted: 4 April 2016/Published online: 13 April 2016

(C) The Association of Bone and Joint Surgeons (B) 2016

\section{History}

In 1938, Bankart and Cantab [2] initially described injury to the anteroinferior glenoid labrum. It was not until 1985 that Andrews and colleagues [1] first identified superior glenoid labral disorders by describing the classic mechanism of injury and biomechanics of labral tears through arthroscopic evaluation of 73 elite overhead-throwing athletes. In 1990, Snyder et al. [22] published a retrospective review of 700 shoulder arthroscopies, with diagnosis of 27 superior labral injuries in a cohort of throwing athletes. Their 1990 landmark article [22] established the acronym "SLAP" (superior labral tear, anterior to posterior) lesion and presented the first comprehensive classification of four major injury patterns as a cause of pain and instability, particularly in the overhead throwing athlete.

Although the emphasis of this article is to describe the original Snyder classification, continued clinical and diagnostic advancements have given rise to the recognition of variations in complex labral disorders [4]. Numerous subclassification and expanded classification systems have

Each author certifies that he or she, or a member of his or her immediate family, has no funding or commercial associations (eg, consultancies, stock ownership, equity interest, patent/licensing arrangements, etc) that might pose a conflict of interest in connection with the submitted article.

All ICMJE Conflict of Interest Forms for authors and Clinical Orthopaedics and Related Research ${ }^{\mathbb{R}}$ editors and board members are on file with the publication and can be viewed on request.

Z. S. Ahsan (凹), J. E. Hsu, A. O. Gee

Department of Orthopaedics and Sports Medicine, University of

Washington, 325 9th Avenue, Box 359798, Seattle, WA 98104,

USA

e-mail: zahsan@uw.edu been developed based on the original description of SLAP lesions $[14,15,17,25]$. Despite this, reporting of SLAP lesions in the literature using even the original Snyder classification [22] is widely variable and inconsistent, as is the clinical diagnosis, indication for surgical intervention, and surgical treatment [13].

\section{Purpose}

The Snyder classification provides an anatomic description and characterization of injury severity to the superior labrum and biceps anchor. Although diagnosis using the classification system often currently is made preoperatively based on MR images, the original anatomic description was based solely on an arthroscopic diagnosis. Based on these findings, the classification also provides a context for treatment recommendations. In the original description, a torn and frayed labrum was treated with excision or débridement, whereas recommendations of repair versus biceps tenodesis were made for an unstable biceps anchor. This classification system has not been shown to provide any prognostic value in the treatment of these lesions [22].

\section{Description of the Snyder Classification System}

The Snyder classification was first documented in 1990 with four described injury patterns (Types I through IV) in 27 patients (Fig. 1). The original description of the SLAP lesion was made at the time of arthroscopy, and no imaging test at that time was thought to be accurate to diagnose these lesions [22]. Type I lesions are characterized by degenerative fraying of the superior labrum free edge with 


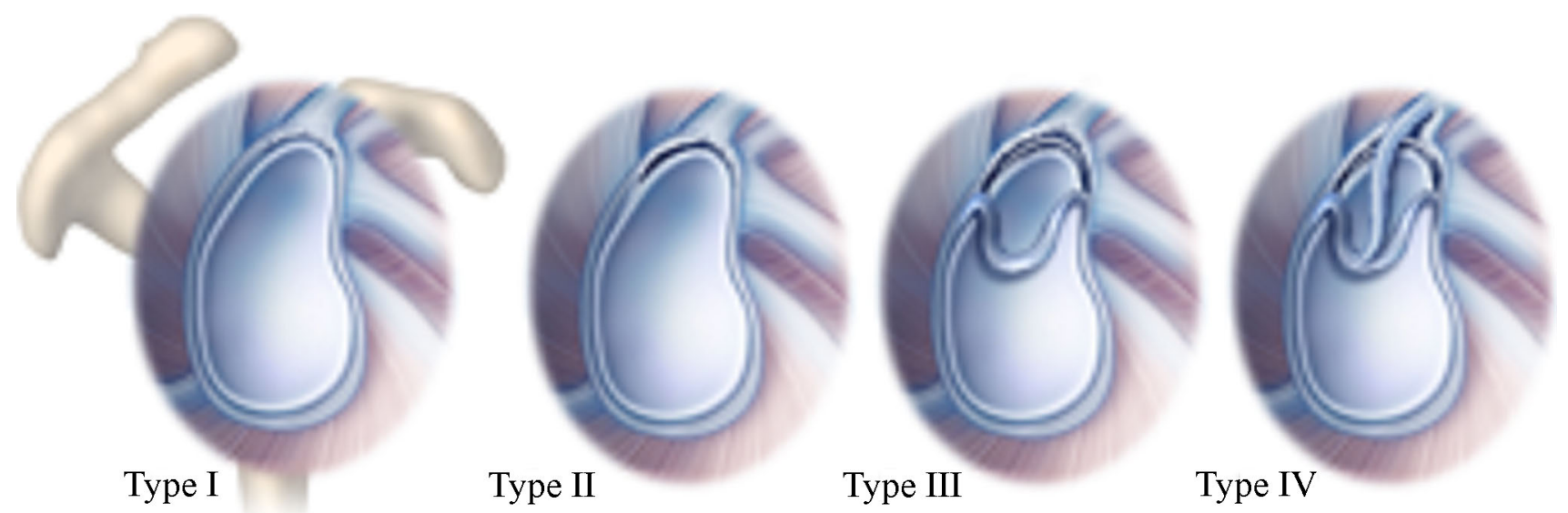

Fig. 1 The expanded classification of SLAP lesions with Types I to IV outlining the original Snyder classification are shown.

intact peripheral attachment and stable biceps tendon anchor. This is notably a common finding in middle-aged and elderly patient populations, suggesting that it may be a degenerative finding that is not a definite source of pain [4, 22]. Type II SLAP tears are described as degenerative fraying with additional detachment of the superior labrum and biceps from the glenoid resulting in an unstable labralbiceps anchor [22]. Type III lesions entail a bucket-handle tear of the superior labrum with an intact biceps tendon anchor [22]. Type IV lesions include a displaced buckethandle labral tear with extension into the biceps tendon root $[4,22]$.

There have been studies that subclassify and expand on the original Snyder description [14, 15, 17]. Morgan et al. [15] further subclassified Type II SLAP lesions based on their location owing to discrete clinical and anatomic features. A retrospective review of 102 Type II SLAP lesions established distinct categories of anterior (37\%) (Type IIA), posterior (31\%) (Type IIB), and combined anterior and posterior (31\%) (Type IIC) lesions [15].

An additional three types of lesions were described by Maffet et al. [14] to further characterize combined lesions rendering the shoulder unstable [3]. These are: a Bankarttype labral disruption, which extends superiorly in continuity with a Type II SLAP lesion (Type V); an unstable flap tear of the labrum in conjunction with a Type II SLAP lesion (Type VI); and a superior labrum and biceps tendon separation that extends anteriorly through the capsule, inferior to the middle glenohumeral ligament (Type VII) [4, 10]. Powell et al. [17] further expanded the classification to include another three variants of Type II SLAP lesions: Type II with extension into the posterior labrum (Type VIII); Type II tears with circumferential labral disruption (Type IX); and Type II lesions combined with posteroinferior labral disruption (Type X) $[4,17]$.

\section{Validation}

Validation of the Snyder SLAP tear classification system has been investigated in multiple studies that document low to moderate interobserver reproducibility using direct observation during arthroscopy (Table 1). The earliest such study reported the interobserver reliability of orthopaedic surgeons in evaluating intraarticular structures involved in real-time diagnostic shoulder arthroscopy by videotape in patients with shoulder instability [19]. Sasyniuk et al. [19] evaluated reliability via percent agreement among observers via statistical analysis although they had a limited sample size of 20 patients. The pathologic distinction in the superior labrum was only to discern normal or abnormal and the specific lesion of the Snyder SLAP classification was not addressed. They concluded that interobserver reliability for orthopaedic shoulder arthroscopic assessment was poor overall $(<40 \%)$ and approximately $60 \%$ to $70 \%$ in focally evaluating superior labrum lesions.

In 2008, Gobezie et al. [8] conducted a statistical analysis of the reliability of the Snyder classification. A group of 73 surgeons (68\% fellowship-trained in arthroscopy, $51 \%$ with $10+$ years in practice) assessed 22 videos to determine the type of SLAP lesion (Snyder classification) and the type of treatment to ascribe to each scenario. Interobserver reliability showed $68 \%$ to $81 \%$ agreement in terms of diagnosis $(\kappa=0.75)$ using the Snyder classification. Intraobserver reliability showed low agreement for native anatomy through Type IV SLAP lesions $(\kappa=0.54)$ among 17 surgeons who responded to the secondary survey. Despite sample size and response rate as limitations of the study, Gobezie et al. [8] concluded that overall interobserver variability showed that shoulder arthroscopists had difficulty distinguishing Types I and II SLAP tears and less than $50 \%$ of surgeons agreed on the diagnosis and 
Table 1. Video studies assessing diagnosis of SLAP lesions

\begin{tabular}{|c|c|c|c|c|c|c|}
\hline Study & $\begin{array}{l}\text { Year } \\
\text { published }\end{array}$ & $\begin{array}{l}\text { Number of reviewing } \\
\text { surgeons }\end{array}$ & $\begin{array}{l}\text { Number of } \\
\text { videos }\end{array}$ & $\begin{array}{l}\text { Method of } \\
\text { comparison }\end{array}$ & $\begin{array}{l}\text { Interobserver } \\
\text { reliability }\end{array}$ & $\begin{array}{l}\text { Intraobserver } \\
\text { reliability }\end{array}$ \\
\hline $\begin{array}{l}\text { Gobezie et al. } \\
\text { [8] }\end{array}$ & 2008 & 73 & 22 & Arthroscopy video & $\kappa=0.75$ & $\kappa=0.54$ \\
\hline Jia et al. [11] & 2011 & 5 & 90 & Arthroscopy video & $\kappa=0.80$ & $\kappa=0.67$ \\
\hline Wolf et al. [25] & 2011 & 11 & 50 & Arthroscopy video & $\kappa=0.39$ & $\kappa=0.47$ \\
\hline
\end{tabular}

SLAP $=$ superior labrum anterior and posterior.

treatment of Types II and III lesions, respectively. Notably, the interobserver variability in their study improved when the diagnosis was made based on the treatment that would be recommended for the injury ( $\kappa=0.95$ for no treatment, $\kappa=0.75$ for debridement of labrum, $\kappa=0.68$ for labral repair, and $\kappa=0.32$ for repair of labrum and/or biceps tenodesis).

Wolf et al., in 2011, [25] performed a cohort study with 11 surgeons viewing a total of 50 shoulder arthroscopy videos a total of three times; initially without additional information, a second time in a new order with an additional clinical vignette, and the third time after a duration of 8 months without clinical vignette. Overall interobserver reliability and intraobserver reliability were moderate at best $(\kappa=0.39$ to $\kappa=0.47)$. In a surprisingly high number of cases, surgeons changed the classification for the superior labrum between viewings. Twenty-eight percent of cases had a change in classification, and $36 \%$ had a change in treatment at two separate viewings of identical videos without vignettes [25].

In 2011, Jia et al. [11] conducted an analysis of experienced fellowship-trained shoulder surgeons performing more than 305 shoulder arthroscopies annually. A group of five examiners evaluated 90 videotapes of shoulder arthroscopies performed for SLAP lesions to identify lesion type. This was repeated at least 2 months from the initial evaluation. Jia et al. [11] tested the original Snyder classification (Types I-IV; interobserver variability $\kappa=0.80$ and intraobserver reliability $\kappa=0.67$ ) and two modifications of the Snyder classification in an attempt to improve the reliability between interpreters. The first modification split the SLAP injuries into normal (normal and Type I) and abnormal (Types II-IV) with interobserver variability $\kappa=0.65$ and intraobserver reliability $\kappa=0.79$. The second grouping entailed subclassification of Type II lesions into $\mathrm{A}, \mathrm{B}$, and $\mathrm{C}$ variants as described by Morgan et al. [15], with interobserver variability $\kappa=0.80$ and intraobserver reliability $\kappa=0.60$. Ultimately, Jia et al. [11] determined that among shoulder specialists there was moderate reliability using the Snyder classification for distinguishing SLAP lesions. Modifications to the classification regarding normal versus abnormal led to increased intraobserver reliability ( $\kappa=0.67$ to $\kappa=0.79$, respectively) whereas interobserver reliability remained similar $(\kappa=0.65$ to $\kappa=$ 0.67 , respectively). This attempt to simplify the classification helped improve intraobserver reliability to near perfect, although the reason why interobserver agreement remained unchanged might be attributable to the openended nature of their study questionnaire.

\section{Limitations}

Classification of SLAP tears using the original Snyder description lacks adequate reproducibility. This may be partly attributable to the difficulty in understanding even normal superior labral anatomy and age-related changes that can occur [20]. The presence of a sublabral sulcus is not considered abnormal, yet our understanding regarding how to differentiate a normal sublabral foramen from a SLAP lesion is not well characterized or described. As Snyder et al. [22] noted in their original description referencing Type II SLAP lesions, "the question arises whether this is indeed pathologic or simply a variation of normal anatomy." Furthermore, determining whether a SLAP lesion is a source of clinical symptoms can be challenging. In the original series describing the Snyder classification [22], a large proportion of the original 27 patients were found to have concomitant shoulder disorders, and it is possible that the observed superior labral disorder could have been an incidental finding. A subsequent study showed a high prevalence of SLAP tears in asymptomatic middle-aged individuals [20].

Given the difficulties in reliably classifying SLAP lesions based on arthroscopic videos [8], it is not surprising that physical examination maneuvers [5, 18] and MRI findings [21] are reported to be unreliable in correctly diagnosing SLAP lesions. In two systematic reviews of physical examination reliability and validity for diagnostic accuracy of SLAP tears [5, 12], the methodologically robust studies determined that Speed's and Yergason's tests (among all maneuvers) showed sensitivity of 32\% and 
$43 \%$ and specificity of $79 \%$ and $75 \%$, respectively $[5,12]$. The confidence interval for likelihood ratios all included 1.0, indicating that Speed's and Yergason's tests could not rule in or rule out a SLAP lesion in comparison to arthroscopy [12]. Studies investigating MRI and MR arthrography in diagnosing SLAP tears have reported sensitivity and specificity ranging from $66 \%$ to $98 \%$ and $13 \%$ to $89 \%$, respectively $[6,7,9,20,21,23]$. Variation in study design, MRI methods, and lack of reliability among observers likely accounts for such a wide discrepancy [16].

The overarching theme regarding the Snyder classification of SLAP lesions is that interobserver variability shows only moderate agreement for the diagnosis of SLAP tears using any modality, indicating that the classification and its expansions are not readily reproducible. Without a reliable method of classifying these lesions and correlating them to clinical symptomatology, clinicians should question the lack of uniformity for surgical indications [13] and the ever-increasing incidence of SLAP repair surgery [24].

\section{Conclusions}

The original Snyder classification has provided a basis for anatomic description of superior labral lesions. However, validation studies have not shown the classification to be reliable. Challenges in correlating clinical symptoms with MRI and physical examination findings make appropriate surgical indications and surgical interventions difficult to establish.

\section{References}

1. Andrews JR, Carson WG Jr, McLeod WD. Glenoid labrum tears related to the long head of the biceps. Am J Sports Med. 1985;13:337-341.

2. Bankart AS, Cantab MC. Recurrent or habitual dislocation of the shoulder-joint. 1923. Clin Orthop Relat Res. 1993;291:3-6.

3. Barber FA, Field LD, Ryu R. Biceps tendon and superior labrum injuries: decision-making. J Bone Joint Surg Am. 2007;89:18441855.

4. Bedi A, Allen AA. Superior labral lesions anterior to posterior: evaluation and arthroscopic management. Clin Sports Med. 2008;27:607-630.

5. Calvert E, Chambers GK, Regan W, Hawkins RH, Leith JM. Special physical examination tests for superior labrum anterior posterior shoulder tears are clinically limited and invalid: a diagnostic systematic review. J Clin Epidemiol. 2009;62:558563.

6. Connell DA, Potter HG, Wickiewicz TL, Altchek DW, Warren RF. Noncontrast magnetic resonance imaging of superior labral lesions: 102 cases confirmed at arthroscopic surgery. Am J Sports Med. 1999;27:208-213.

7. Dinauer PA, Flemming DJ, Murphy KP, Doukas WC. Diagnosis of superior labral lesions: comparison of noncontrast MRI with indirect MR arthrography in unexercised shoulders. Skeletal Radiol. 2007;36:195-202.

8. Gobezie R, Zurakowski D, Lavery K, Millett PJ, Cole BJ, Warner JJP. Analysis of interobserver and intraobserver variability in the diagnosis and treatment of SLAP tears using the Snyder classification. Am J Sports Med. 2008;36:1373-1379.

9. Herold T, Hente R, Zorger N, Finkenzeller T, Feuerbach S, Lenhart M, Paetzel C. [Indirect MR-arthrography of the shoulder: value in the detection of SLAP-lesions][in German]. Rofo. 2003;175:1508-1514.

10. Higgins LD, Warner JJ. Superior labral lesions: anatomy, pathology, and treatment. Clin Orthop Relat Res. 2001;390:7382.

11. Jia X, Yokota A, McCarty EC, Nicholson GP, Weber SC, McMahon PJ, Dunn WR, McFarland EG. Reproducibility and reliability of the Snyder classification of superior labral anterior posterior lesions among shoulder surgeons. Am J Sports Med. 2011;39:986-991.

12. Karlsson J. Physical examination tests are not valid for diagnosing SLAP tears: a review. Clin J Sport Med. 2010;20:134135.

13. Kibler WB, Sciascia A. Current practice for the diagnosis of a SLAP lesion: systematic review and physician survey. Arthroscopy. 2015;31:2456-2469.

14. Maffet MW, Gartsman GM, Moseley B. Superior labrum-biceps tendon complex lesions of the shoulder. Am J Sports Med. 1995;23:93-98.

15. Morgan CD, Burkhart SS, Palmeri M, Gillespie M. Type II SLAP lesions: three subtypes and their relationships to superior instability and rotator cuff tears. Arthroscopy. 1998;14:553-565.

16. Phillips JC, Cook C, Beaty S, Kissenberth MJ, Siffri P, Hawkins RJ. Validity of noncontrast magnetic resonance imaging in diagnosing superior labrum anterior-posterior tears. J Shoulder Elbow Surg. 2013;22:3-8.

17. Powell SE, Nord KD, Ryu RK. The diagnosis, classification, and treatment of SLAP lesions. Oper Tech Sports Med. 2004;12:99110 .

18. Sandrey MA. Special physical examination tests for superior labrum anterior-posterior shoulder tears: an examination of clinical usefulness. J Athl Train. 2013;48:856-858.

19. Sasyniuk TM, Mohtadi NG, Hollinshead RM, Russell ML, Fick GH. The inter-rater reliability of shoulder arthroscopy. Arthroscopy. 2007;23:971-977.

20. Schwartzberg R, Reuss BL, Burkhart BG, Butterfield M, Wu JY, McLean KW. High prevalence of superior labral tears diagnosed by MRI in middle-aged patients with asymptomatic shoulders. Orthop J Sports Med. 2016;4:2325967115623212; doi: 10.1177/ 2325967115623212.

21. Sheridan K, Kreulen C, Kim S, Mak W, Lewis K, Marder R. Accuracy of magnetic resonance imaging to diagnose superior labrum anterior-posterior tears. Knee Surg Sports Traumatol Arthrosc. 2015;23:2645-2650.

22. Snyder SJ, Karzel RP, Del Pizzo W, Ferkel RD, Friedman MJ. SLAP lesions of the shoulder. Arthroscopy. 1990;6:274-279.

23. Tung GA, Entzian D, Green A, Brody JM. High-field and lowfield MR imaging of superior glenoid labral tears and associated tendon injuries. AJR Am J Roentgenol. 2000;174:1107-1114.

24. Vogel LA, Moen TC, Macaulay AA, Arons RR, Cadet ER, Ahmad CS, Levine WN. Superior labrum anterior-to-posterior repair incidence: a longitudinal investigation of community and academic databases. J Shoulder Elbow Surg. 2014;23:e119-e126.

25. Wolf BR, Britton CL, Vasconcellos DA, Spencer EE; MOON Shoulder Group. Agreement in the classification and treatment of the superior labrum. Am J Sports Med. 2011;39:2588-2594. 